CARDIOVASCULAR MEDICINE

\title{
Clopidogrel reduces migraine with aura after transcatheter closure of persistent foramen ovale and atrial septal defects
}

\author{
P T Wilmshurst, S Nightingale, K P Walsh, W L Morrison
}

Heart 2005;91:1173-1175. doi: 10.1136/hrt.2004.047746

\begin{abstract}
Objective: To report the clinical events leading to alteration of an anticoagulation regimen for patients undergoing transcatheter closure of an atrial shunt and how this affected migraine symptoms after the closure procedure.

Method: Audit of a change of anticoagulant regimen.

Results: In the first few weeks after a closure procedure migraine frequency and severity increased despite treatment with aspirin for six months in 71 patients. Severe attacks of migraine with aura, including status migrainosus, in the first few weeks after transcatheter closure were terminated by addition of clopidogrel to aspirin treatment. Therefore, the anticoagulant regimen was changed with addition of clopidogrel for the first month after the closure procedure (90 procedures in 89 patients). Fewer patients had migraine with aura in the first month after transcatheter closure when taking the combination of clopidogrel and aspirin compared with aspirin alone (11 of $90(12.2 \%)$ v 30 of $71(42.3 \%), p<0.001)$. Episodes of migraine with aura were more severe and more frequent in patients taking aspirin alone. Conclusion: The combination of clopidogrel for four weeks and aspirin for six months is superior to aspirin alone for six months for preventing migraine with aura after transcatheter closure of an atrial shunt. This beneficial effect of a powerful inhibitor of platelet aggregation suggests that platelets may have a role in pathogenesis of migraine. This may be because of an effect on serotonin stores. Whether clopidogrel has a role in treatment of migraine in other clinical situations requires investigation.
\end{abstract}

See end of article for authors' affiliations

Correspondence to: Dr Peter T Wilmshurst, Royal Shrewsbury Hospital, Shrewsbury SY3 8XQ, UK; peter wilmshurst@rsh.nhs.uk

3 October 2004

$M$ igraine with aura and atrial right to left shunts (persistent foramen ovale and atrial septal defects) are associated..$^{1-3}$ We postulated that the relation may be causal if substances that trigger migraine, such as vasoactive chemicals or microemboli, can circumvent the pulmonary filter by passage through the cardiac shunt to reach the brain. ${ }^{3}$ Observational studies suggest that transcatheter closure of persistent foramen ovale and atrial septal defects, in divers who have had decompression illness and patients with paradoxical thromboembolism, reduces frequency and severity of migraine symptoms (migraine headache or aura or both) in the long term. ${ }^{4-6}$ Migraine symptoms, particularly migraine with aura, are often initially exacerbated, however, in the first few weeks after the closure procedure despite treatment with aspirin for six months. ${ }^{4}$ We postulated that the initial exacerbation may be the result of liberation of vasoactive substances or microemboli from thrombus adherent to the left atrial surface of the occluder. ${ }^{4}$

We report the clinical events that caused us to alter our anticoagulation regimen for patients undergoing transcatheter closure of an atrial shunt and how this affected the occurrence of migraine symptoms after the closure procedure.

\section{METHODS}

Migraine symptoms are defined by the Headache Classification Committee of the International Headache Society. ${ }^{7}$ The term "status migrainosus" is not in this classification but we use it in this paper to describe repeated attacks of migraine with little or no intervening period free from aura or migraine headache.

Comparison of the two groups (aspirin treatment alone for six months or aspirin for six months with clopidogrel for the first month) was by $\chi^{2}$ test and $t$ test as appropriate. Numerical data are expressed as mean (SD). A p $<0.05$ was considered significant.

Shropshire research ethics committee approved the audit.

\section{RESULTS}

There is no standard anticoagulant regimen for patients having transcatheter closure of atrial shunts. We initially prescribed aspirin 150-300 mg daily for six months after the closure procedure. Of 71 consecutive patients treated with aspirin alone after a closure procedure to prevent recurrence of decompression illness or stroke as a result of paradoxical embolism or for haemodynamic reasons, 30 experienced in the first month either an exacerbation of their migraine symptoms, consisting of migraine with aura, status migrainosus, or aura alone $(\mathrm{n}=21)$, or new migraine symptoms $(\mathrm{n}=9)$.

Five of the 30 patients had very severe attacks of migraine with aura. We did not alter our anticoagulant regimen, consisting of aspirin alone, for two of the five patients because the patients had passed through the worst of their symptoms by the time they reported them to us. One of these, a woman who had infrequent migraine with aura before the closure procedure, had status migrainosus afterwards. The other was a man who had about 12 attacks of migraine with an aura consisting of hemiplegia and a visual aura after the closure procedure but no history of migraine before the procedure. For the three remaining patients we altered the anticoagulant regimen because of the severity of their ongoing symptoms. Two, who usually had two attacks of migraine with aura a year, had status migrainosus. One of these reported between 40 and 50 cycles of migraine aura and migraine headache within a period of four weeks. The third patient, a woman, who had no history of migraine before the closure procedure, experienced in the two weeks after the procedure four attacks of migraine, which were each associated with fortification spectra, hemianaesthesia, and expressive dysphasia. We were concerned that these severe symptoms may be caused by thrombus formation or liberation of vasoactive substances (such as serotonin) as a result of platelet activation on the left atrial disc and felt that we should intensify the anticoagulant regimen of these three patients. 
In most of our patients we implanted an Amplatzer device. These are Nitinol wire mesh discs interwoven with Dacron. Coronary artery stents also have a wire mesh. The combination of clopidogrel and aspirin is superior to aspirin alone for preventing acute stent thrombosis. ${ }^{8}$ We had already treated two patients who were intolerant of aspirin with clopidogrel $75 \mathrm{mg}$ daily and neither had migraine symptoms after their closure procedure. Therefore, for these three patients with severe symptoms we added clopidogrel for 2-4 weeks to their aspirin. Within 4-24 hours their migraine symptoms had ceased. Two of these three patients had no recurrence of migraine or aura during or at the end of clopidogrel treatment. In the third patient, attacks of migraine with aura restarted four days after stopping clopidogrel and were terminated by restarting clopidogrel for a further two weeks. On stopping the clopidogrel for the second time, the patient had recurrence of mild episodes of migraine with aura. The patient elected to wait to see whether the migraine with aura would abate without restarting clopidogrel, which it did over two weeks.

These observations caused us to change our standard anticoagulant regimen to aspirin for six months plus clopidogrel $75 \mathrm{mg}$ daily for the first month (or longer if clinically indicated) for 90 closure procedures in 89 patients. Two patients, who had significant residual shunts, had a second closure procedure. One took aspirin and clopidogrel on both occasions. The other took aspirin alone after the first closure procedure and clopidogrel and aspirin after the second.

The 90 occasions when clopidogrel and aspirin were given and the 71 historic control periods when aspirin alone was given were not significantly different with respect to number of women (31 of $71(44 \%) v 29$ of $90(32 \%))$ or number with a history of migraine with aura (36 of 71 (51\%) v 50 of 90 $(56 \%))$. Patients given aspirin alone were significantly younger (37.0 (10.5) years) than those given aspirin and clopidogrel (41.7 (10.1) years), but there was no relation between age and occurrence of migraine symptoms before or after the closure procedure.

On the 90 occasions that the combination of clopidogrel and aspirin was given, 11 patients $(12.2 \%)$ reported migraine with aura or aura alone in the month when they were taking clopidogrel and aspirin. In every case the symptoms were mild, brief, and infrequent. Eight of the 11 had a history of migraine with aura before the closure procedure. The number of patients who got migraine with aura or aura alone during the equivalent month when they were taking aspirin alone was 30 of $71(42.3 \%)$ and the difference was significant $(p<0.001)$. Twenty one of the 30 had a history of migraine with aura before the closure procedure. Episodes of migraine were more severe and frequent in individual patients taking aspirin alone than in those taking clopidogrel and aspirin. No patient taking the combination of clopidogrel and aspirin experienced severe symptoms, such as those described above in the five patients taking aspirin alone.

Sixteen patients, who had taken clopidogrel and aspirin, reported that in the week after stopping the clopidogrel, they experienced migraine with aura or aura alone though they were still taking aspirin. Only one of the 16 had migraine symptoms during the month when clopidogrel was taken. One patient, who had no migraine symptoms when taking clopidogrel, had six attacks of migraine with aura in short succession after stopping the drug. Clopidogrel was then restarted for a further four weeks. She had no migraine symptoms during her second period of clopidogrel treatment but on stopping the drug her migraine with aura recurred. She took clopidogrel for a further two weeks with no migraine or aura during that time. Then she stopped taking clopidogrel for the third time with recurrence of migraine with aura, but it was only mild and it settled over a few weeks without treatment and has not recurred since then.
One patient took aspirin alone after her first closure procedure and clopidogrel and aspirin after the second. She had two attacks of migraine with aura in the week after the first closure procedure but no migraine symptoms after the second. One patient took clopidogrel and aspirin after two closure procedures. He had no migraine symptoms when taking clopidogrel and aspirin but had migraine after stopping the clopidogrel on one occasion.

The only significant bleeding complications were in patients taking clopidogrel and aspirin. One had a gastrointestinal haemorrhage one week after the closure procedure but gastroduodenoscopy and colonoscopy were normal. Another patient had a large pelvic haematoma with blood tracking from the femoral vein puncture site. Neither patient required transfusion.

\section{DISCUSSION}

Over half of our patients with large atrial shunts (persistent foramen ovale that had permitted paradoxical embolism and atrial septal defects) had a history of migraine with aura. This is in keeping with high prevalence reported in other studies. ${ }^{1-3}$ Transcatheter closure of the shunts resulted in early exacerbation of migraine symptoms in some and development of migraine with aura in some patients who had not had it before as previously reported. ${ }^{4}$

Our observations were not obtained in a randomised, double blind comparison of two treatment regimens. Nevertheless there was an obvious and large reduction in acute migraine symptoms (migraine with aura, status migrainosus, or aura alone) after transcatheter closure of a persistent foramen ovale or atrial septal defect when patients were given a combination of clopidogrel for four weeks and aspirin for six months compared with aspirin alone for six months. Addition of clopidogrel rapidly aborted status migrainosus in those patients who were taking aspirin alone. These observations may help in managing this group of patients but may also increase our understanding of the pathophysiology of migraine.

Clopidogrel blocks platelet aggregation by interfering with the binding of ADP to its receptor on the platelet surface, an action distinct from that of aspirin. Findings in studies seeking a link between migraine and platelet function are conflicting, but there is some evidence that platelets from migraineurs aggregate more readily than normal in response to exposure to several vasoactive amines, including serotonin and ADP. ${ }^{9}$ Platelets contain most of the serotonin normally present in the blood and at the start of an attack of migraine there is a significant rise in plasma serotonin and urinary excretion of 5-hydroxyindoleacetic acid, a breakdown product. ${ }^{9}$ Serotonin is involved in the pathogenesis of an attack of migraine but its precise role is unclear. Drugs with high affinities for specific serotonin receptors have an important role in management of migraine. Serotonin in venous blood does not normally reach the systemic arterial circulation because monoamine oxidase virtually totally destroys serotonin in its first passage through lung tissue. ${ }^{10}$ Serotonin may reach systemic arteries if a large amount is released so that the ability of lung tissue to metabolise the serotonin is overwhelmed, if the concentrations of lung monoamine oxidase are low, if there is a right to left shunt that allows the lung tissue to be bypassed, or if platelets aggregate in the left side of the heart as may occur if platelets are activated on the left atrial disc of an occlusion device.

Our observations may require confirmation in a blind, placebo controlled comparison of anticoagulant regimens in patients having transcatheter closure of atrial shunts. It is clear that the aetiology of migraine is complex. Not all migraineurs respond to treatment with antiplatelet drugs and many effective antimigraine treatments have no known 
antiplatelet actions. However, these observations suggest that platelets may have a role in migraine aetiology in some circumstances. We believe that the possible beneficial effects of clopidogrel in migraine should be investigated, even in the absence of a cardiac shunt.

\section{Authors' affiliations \\ P T Wilmshurst, S Nightingale, Royal Shrewsbury Hospital, Shrewsbury, UK}

K P Walsh, Our Lady's Hospital for Sick Children, Dublin, Republic of Ireland

W L Morrison, The Cardiothoracic Centre, Liverpool, UK

Conflicts of interest: None

All authors participated in the clinical care of the patients, the decision to add clopidogrel to aspirin as an anticoagulant, analysing the data, and writing the report.

\section{REFERENCES}

1 Del Sette M, Angeli S, Leandri M, et al. Migraine with aura and right-to-left shunt on transcranial Doppler: a case-control study. Cerebrovasc Dis 1998;8:327-30.
2 Anzola GP, Magoni M, Guindani M, et al. Potential source of cerebral embolism in migraine with aura: a transcranial Doppler study. Neurology 1999;52:1622-5

3 Wilmshurst P, Nightingale S. Relationship between migraine and cardiac and pulmonary right-to-left shunts. Clin Sci 2001;100:215-20.

4 Wilmshurst PT, Nightingale S, Walsh KP, et al. Effect on migraine of closure of cardiac right-to-left shunts to prevent recurrence of decompression illness or stroke or for haemodynamic reasons. Lancet 2000;356:1648-51

5 Post MC, Thijs V, Herroelen L, et al. Closure of a patent foramen ovale is associated with a decrease in prevalence of migraine. Neurology 2004;62:1439-40.

6 Schwerzmann M, Wiher S, Nedeltchev K, et al. Percutaneous closure of patent foramen ovale reduces the frequency of migraine attacks. Neurology 2004;62:1399-401

7 Anon. Headache Classification Committee of the International Headache Society. Classification and diagnostic criteria for headache disorders, cranial neuralgias and facial pain. Cephalalgia 1988;8(suppl 7):1-96.

8 Anon. CURE Study Investigators. The Clopidogrel in unstable angina to prevent recurrent events (CURE) trial programme: rationale, design and baseline characteristics including a meta-analysis of the effects of thienopyridines in vascular disease, Eur Heart J 2000;21:2033-41.

9 Bradley WG, Daroff RB, Fenichel GM, et al. Neurology in clinical practice, 3rd ed. Boston: Butterworth-Heinemann, 2000:1852.

10 Gaddum JH, Hebb CO, Silver A, et al. 5-Hydroxytryptamine: pharmacological action and destruction in perfused lungs. $Q J$ Exp Physiol 1953;38:255-62.

\section{IMAGES IN CARDIOLOGY}

\section{Multiple thrombi in the left atrium}

A 76 year old woman was referred to our cardiology department for percutaneous mitral commissurotomy. Two months before her admission, she underwent emergency vascular surgery for leg ischaemia successfully. She was in atrial fibrillation and a severe mitral stenosis was diagnosed (mitral valve area $1 \mathrm{~cm}^{2}$, mean transmitral gradient $12 \mathrm{~mm} \mathrm{Hg}$ ). She received two months of oral anticoagulation with adequate international normalised ratio (INR) range. Before the procedure, transoesophageal echocardiography was performed. At least four separate thrombi were identified: one in the left appendage which was almost occluded (panel A), a smaller one, very mobile, close to the left appendage (panel $\mathrm{B})$, a large one $(16 \times 26 \mathrm{~mm})$ on the anterior side of the atrium near the aorta (panel C), and a very small one on the left side of the interatrial septum (panel D). Just after transoesophageal echocardiography the patient developed a stroke with complete left hemiplegia. Since she was already on oral anticoagulation with an INR $>2$, and systemic thrombolysis was contraindicated, in situ fibrinolysis was performed. Unfortunately, a new cerebral embolic event occurred and the patient died two days later.

Left atrial thrombi are common complications in mitral stenosis but are usually unique and observed in the left appendage. The present case illustrates the high risk of embolic event in mitral stenosis that is not completely discarded despite adequate anticoagulation. The most efficient treatment remains an earlier management of the stenosis by percutaneous commissurotomy or surgery.

D Messika-Zeitoun A Nicoud

A Vahanian david.messika-zeitoun@bch.ap-hop-paris.fr

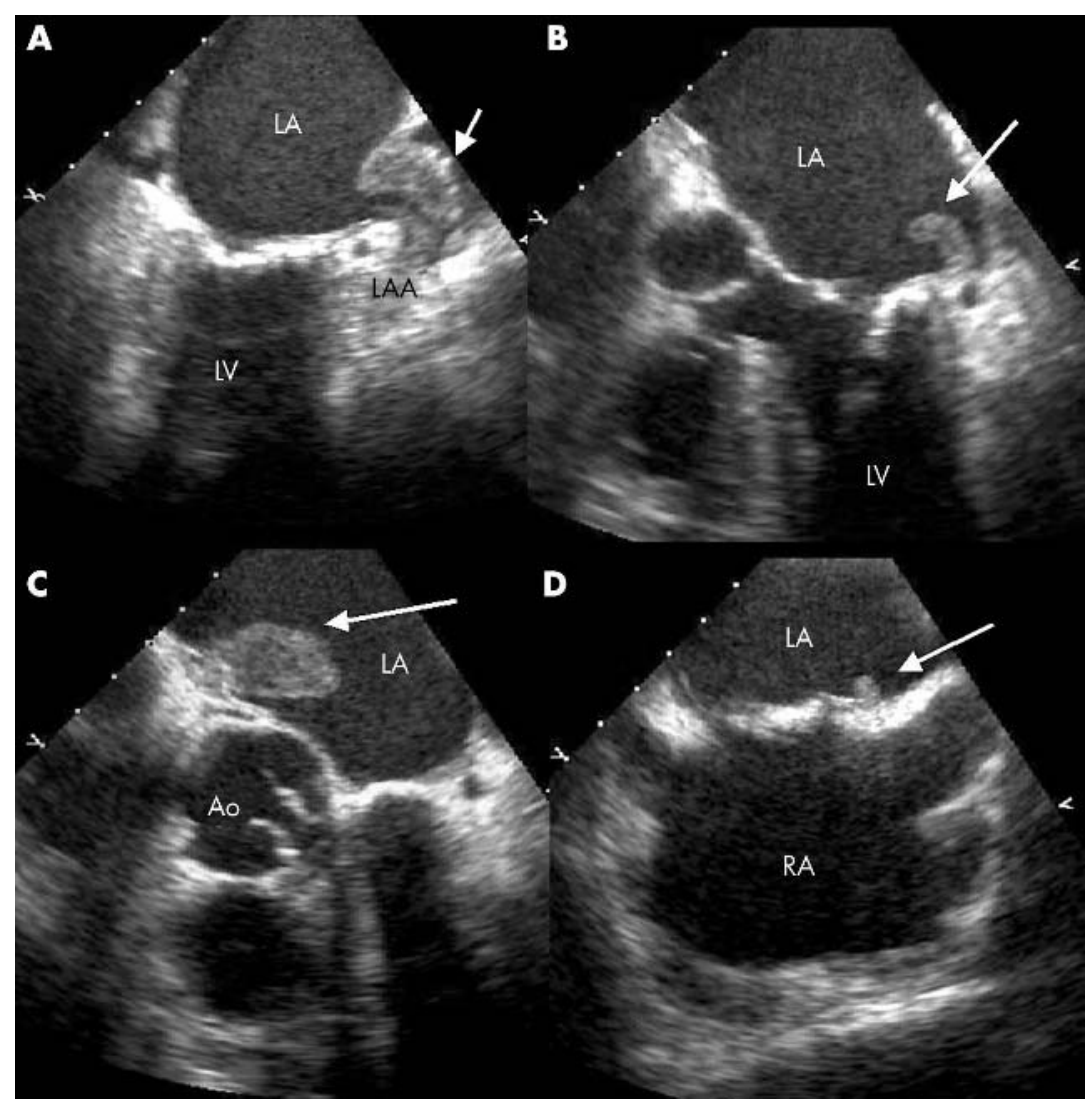

